\title{
The "Great Masquerader" Strikes Again: Secondary Syphilis Presentation with Erythema Multiforme (EM)-Like Lesions
}

\author{
Adi Brom, Idan Goren, Narin Nard Carmel, Gad Segal
}

Sackler Faculty of Medicine, Tel Aviv University, Tel Aviv, Israel

Sheba Medical Center, Tel Hashomer, Ramat Gan, Israel

\begin{abstract}
Syphilis is a sexually transmitted disease known to present with highly variable manifestations, especially when left untreated. Patients who present to Internal Medicine Departments with fever and a rash are always a diagnostic challenge since mild viral diseases and life-threatening bacterial infections may manifest themselves similarly. In the following case presentation, we describe a patient with 1 month's duration of fever and rash on the palms of the hand and soles of the feet, in the form of erythema multiforme (EM)-like lesions. His disease was diagnosed as secondary syphilis, once again justifying its name: the "great masquerader".
\end{abstract}

Keywords: Syphilis, erythema multiforme, fever and rash, fever of unknown origin

Received: $30 / 10 / 2014$

Accepted: $20 / 11 / 2014$

Published: 05/12/2014

How to cite this article: Brom A, Goren I, Nard Carmel N, Segal G. The "Great Masquerader" Strikes Again: Secondary Syphilis Presentation with Erythema Multiforme (EM)-Like Lesions. EJCRIM 2014;1:doi: 10.12890/2014_000161

Conflicts of Interests: The authors declare that they have no conflicts of interest related to this research.

This article is licensed under a Commons Attribution Non-Commercial 4.0 License

\section{Introduction}

Syphilis is a sexually transmitted disease known to present with highly variable manifestations, especially when left untreated. Patients who present to Internal Medicine Departments with fever and a rash are always a diagnostic challenge since mild viral diseases and life-threatening bacterial infections may manifest themselves similarly. In the following case presentation, we describe a patient with 1 month's duration of fever and rash on the palms of the hand 
and soles of the feet, in the form of erythema multiforme (EM)-like lesions. His disease was diagnosed as secondary syphilis, once again justifying its name: the "great masquerader".

\section{Case presentation}

A 42-year-old male having sex with a male (MHSM) was admitted to an internal medicine department due to agonizing rectal and scrotal pain for a week prior to admission. He presented with a 1-month history of throat pain and occasional fever, mostly at nighttime. At the beginning of his illness, he noticed painful aphthous ulcers in the oral cavity and peri-oral lesions he recognized as herpes; these had resolved spontaneously a few days previously. Prior to admission, he received several courses of various antibiotics from his family physician without any response or improvement. Four days prior to admission, a painful, vesicular rash appeared on his penis and scrotum without adequate response to topical antibiotic creams. He had no other relevant medical history; he denied taking new medications or illicit drug use. There were no urinary complaints, penile discharge, arthralgia or pruritus.

Upon admission, his vital signs were within normal limits. The patient appeared to be a healthy young man. Physical examination revealed exudates on the pharyngeal tonsils bilaterally and a raised, white lesion on the tongue, without any ulcerative lesions in the oral cavity. Target-like lesions were present bilaterally on the palms of the hands and soles of the feet, which were defined by a dermatologist as EM-like lesions (Fig. 1). Scrotal lesions were consistent with herpes simplex infection. Several perianal lesions were present, consistent with condylomata lata.

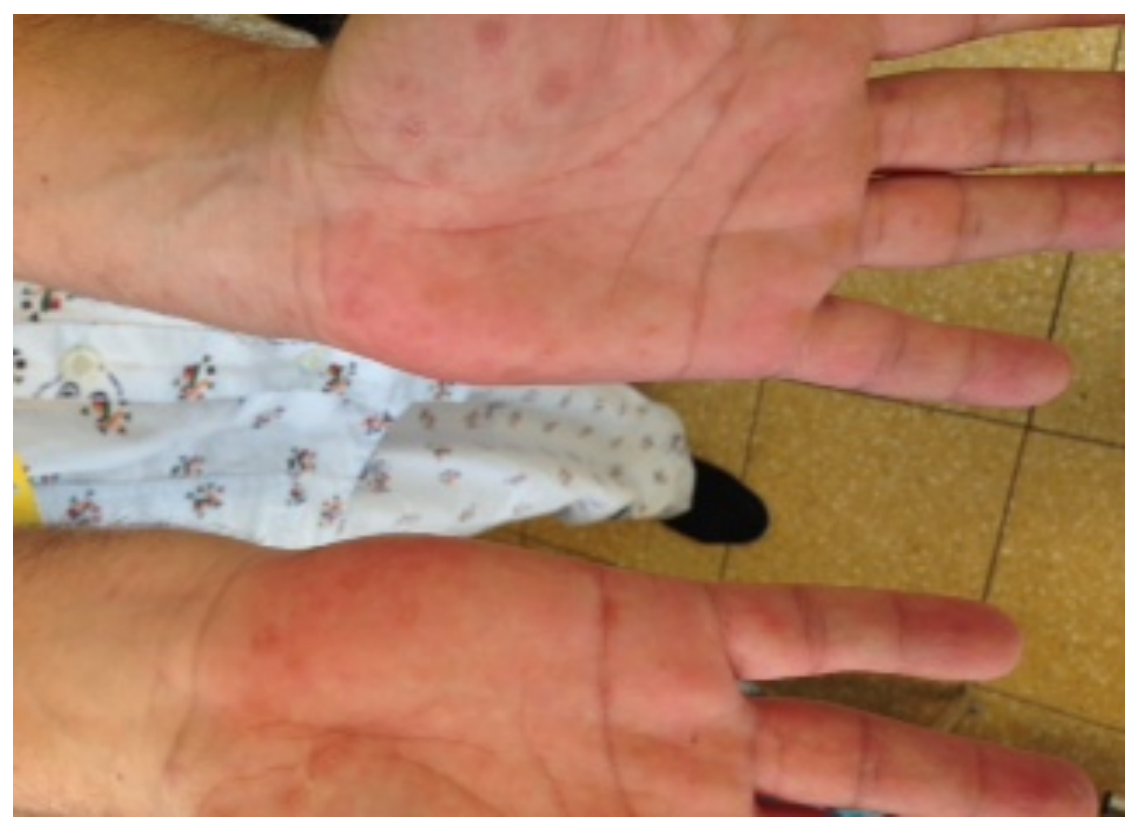

On initial laboratory workup, a complete blood count showed mild leukocytosis. Blood chemistry showed elevated alanine transaminase (ALT) and aspartate transaminase (AST) levels with predominantly elevated alkaline phosphatase blood concentration. Serologic tests for hepatitis B and $C$ viruses were negative, as were HIV-ELISA and polymerase chain reaction (PCR). A rapid plasma reagent (RPR) was positive, with a titre of $1: 16$, and the syphilis immunoassay result was positive as well. PCR from the scrotal lesions was positive for Herpes simplex type 2 .

Figure 1: Erythema multiforme (EM)-like lesions

The patient was diagnosed with secondary syphilis with probable herpetic co-infection. He was treated with penicillin, ceftriaxone and doxycycline with improvement, as well as acyclovir for herpetic infection. He continued his out-patient follow-up in the infectious diseases unit with complete resolution of both rash and fever. 


\section{Discussion}

The hallmarks of secondary syphilis are prominent muco-cutaneous findings, usually presenting as maculo-papular rash, oral mucous patches, patchy scalp alopecia and condylomata lata [1]. Dermatologic manifestations of secondary syphilis can be highly challenging, also presenting as EM-like eruptions, which may represent an immune response to Treponema pallidum. EM is an acute skin condition that is considered to be a type IV hypersensitivity reaction. The differential diagnosis of EM accompanying a febrile illness is extremely wide. Because this condition may be related to a persistent antigenic stimulus, recurrence is very common. It also commonly appears as a result of a drug reaction, mainly to barbiturates, penicillin, phenytoin and sulphonamides. The differential diagnosis for the combination of EM and fever includes infections such as Mycoplasma pneumonia, Bacillus, Lyme disease, histoplasmosis and Epstein-Barr virus. It is also rarely associated with hepatitis $C$ virus infection. Parasites such as Trichomonas species and Toxoplasma gondii may also induce target-like lesions and EM. Possible autoimmune conditions resulting in EM and fever include serum sickness, collagen vascular diseases, necrotizing vasculitis and Behçet's disease. It might also be a precipitating sign of a non-Hodgkin's lymphoma [2, 3]. EM has been previously described as a rare presenting symptom of secondary syphilis in both adults and children [4, 5].

Syphilis is a primarily sexually transmitted disease, caused by the spirochete $T$. pallidum. The incidence of both primary and secondary syphilis has increased in the past decade, with men having sex with men (MHSM) being particularly affected. In this group, the disease is frequently associated with promiscuous behaviour, unprotected anal intercourse and substance abuse [6-8]. The classic presentation of primary syphilis is a solitary non-tender genital chancre, which represents the $T$. pallidum inoculation site and usually appears 10-90 days after infection. Untreated primary disease develops into secondary syphilis within 6-8 weeks. The secondary infection represents widespread dissemination of $T$. pallidum and its clinical presentation is highly varied; some evidence of systemic involvement is seen in the majority of patients, such as headache, malaise, fever, lymphadenopathy or sore throat [1]. Syphilitic hepatitis, as in the case of the current patient, is characterized by a predominantly high alkaline phosphatase level with normal or mildly elevated ALT and AST [9].

Left untreated, primary- or secondary-stage disease might progress into the latent stage of disease, characterized by the absence of symptoms. Months to years later, tertiary syphilis may emerge, characterized by a persistent low-level burden of pathogens, against which a self-destructive immune response is mounted [10]. Tertiary syphilis may presents as neuro-syphilis, cardiovascular syphilis or late benign syphilis.

Diagnostic techniques include both specific and non-specific Treponema testing; patients suspected of having syphilis are usually screened with non-specific Treponema tests, including the venereal disease research laboratory (VDRL) and rapid plasma reagin (RPR) tests. Cases with positive results should undergo specific Treponema testing to confirm the diagnosis, such as with a fluorescent Treponema antibody absorption assay or a $T$. pallidum particle agglutination test [11].

Co-infection with HIV is common given the common epidemiologic risk factors, and is reported to be as high as 50\% [6]. Therefore, HIV testing has an important role. Acceptable therapy is intra-muscular penicillin, and additional antibiotics in case of co-infection. With timely treatment, patients with secondary syphilis have a good prognosis since $T$. pallidum remains extremely sensitive to penicillin [12]. The Jarisch-Herxheimer reaction is a possible adverse effect amongst 
patients treated for secondary syphilis, consisting of acute exacerbation of symptoms due to massive cytokine release . Treatment adequacy should be tracked by repeating the VDRL test at 3-, 6- and 12-month intervals until Treponema becomes undetectable.

\section{Conclusion}

Syphilis is a treatable infectious disease that should be tested for in the investigation of sexually active patients with fever and rash in order to prevent late life-threatening complications. EM is an uncommon presenting sign of secondary syphilis.

\section{Learning Points}

- Syphilis continues to be a relevant and viable option in each and every workup of "fever and rash".

- Secondary syphilis should be included in the list of differential diagnoses for EM -like skin lesions.

- Timely identification and treatment of secondary syphilis is valuable for successful, definitive therapy.

\section{References}

1. Read PJ, Donovan B. Clinical aspects of adult syphilis, Intern. Med. J. 2012;42:614-620.

2. Sokumbi O, Wetter DA. Clinical features, diagnosis, and treatment of erythema multiforme: a review for the practicing dermatologist, Int. J. Dermatol. 2012;51:889-902.

3. Lamoreux MR, Sternbach MR, Hsu WT. Erythema multiforme, Am. Fam. Physician 2006;74:1883-1888.

4. Bhate C, Tajirian AL, Kapila R, Lambert WC, Schwartz RA. Secondary syphilis resembling erythema multiforme, Int. J. Dermatol. 2010;49:1321-1324.

5. Wu CC, Tsai CN, Wong WR, Hong HS, Chuang YH. Early congenital syphilis and erythema multiforme-like bullous targetoid lesions in a 1-day-old newborn: detection of Treponema pallidum genomic DNA from the targetoid plaque using nested polymerase chain reaction, J. Am. Acad. Dermatol. 2006;55:S11-15.

6. Mattei PL, Beachkofsky TM, Gilson RT, Wisco OJ. Syphilis: a reemerging infection, Am. Fam. Physician 2012;86:433-440.

7. Brosh-Nissimov T, Mor Z, Avramovich E, Katchman E, Avidor B, Mor O et al. Syphilis outbreak among men who have sex with men, Tel Aviv, Israel, 2008-2009, Isr. Med. Assoc. J. 2012;14:152-156.

8. Champenois K, Cousien A, Ndiaye B, Soukouna Y, Baclet V, Alcarazl et al. Risk factors for syphilis infection in men who have sex with men: results of a case-control study in Lille, France, Sex. Transm. Infect. 2013;89:128-132.

9. Young MF, Sanowski RA, Manne RA. Syphilitic hepatitis, J. Clin. Gastroenterol. 1992;15:174.

10. Kent ME, Romanelli F. Reexamining syphilis: an update on epidemiology, clinical manifestations, and management, Ann. Pharmacother. 2008;42:226-236. 


\section{European Journal}

of Case Reports in

Internal Medicine

11. Tucker JD, Bu J, Brown LB, Yin YP, Chen XS, Cohen MS. Accelerating world wide syphilis screening through rapid testing: a systematic review, Lancet Infect. Dis. 2010;10:381-386.

12. Cohen SE, Klausner JD, Engelman J, Philip S. Syphilis in the modern era: an update for physicians, Infect. Dis. Clin. North Am. 2013;27:705-722. 